\title{
Pediatric Lobular Capillary Hemangioma Nasal Cavity
}

\author{
${ }^{1}$ Saurabh Varshney, ${ }^{2}$ Manu Malhotra, ${ }^{3}$ Navjot Kaur, ${ }^{4}$ Priyanka Gairola
}

\begin{abstract}
A lobular capillary hemangioma $(\mathrm{LCH})$ is a benign uncommon entity of unknown etiology among vascular lesions of the head and neck. Although it has no predilection for age, it is more common in the third decade and in females. Lobular capillary hemangioma usually involves the gingiva, lips, tongue and buccal mucosa. The nasal cavity is a rare location. The most common symptoms are nasal obstruction and epistaxis. The treatment is nasal endoscopic surgery.

We review these vascular lesions and present the clinical features, radiological findings, treatment performed and histopathological results of two cases of $\mathrm{LCH}$ originating in the left inferior turbinate and septum. No recurrence was observed after complete endoscopic resection during 6 months follow-up. Lobular capillary hemangioma should be taken into consideration among the differential diagnosis of a painless hemorrhagic endonasal mass even though it is a rare entity. If complete resection is performed, prognosis is excellent and the recurrence rate is low.
\end{abstract}

Keywords: Capillary hemangioma, Epistaxis, Nasal cavity.

How to cite this article: Varshney S, Malhotra M, Kaur N, Gairola P. Pediatric Lobular Capillary Hemangioma Nasal Cavity. Clin Rhinol An Int J 2015;8(2):76-80.

\section{Source of support: Nil}

Conflict of interest: None

\section{INTRODUCTION}

Lobular capillary hemangioma (LCH), is a benign, rapidly growing lesion, usually solitary which occurs in the skin and mucous membranes of unknown etiology. It was first described as human botryomycosis by Poncet and Dor in 1897. The oral cavity has been reported to be a common site of involvement, while it is rarely located in the nasal cavity. It may be pedunculated or broadbased and can vary in size from a few millimeters to

\footnotetext{
${ }^{1}$ Professor and Head, ${ }^{2}$ Assistant Professor, ${ }^{3,4}$ Senior Resident

${ }^{1}$ Department of Otorhinolaryngology and Head Neck Surgery All India Institute of Medical Sciences, Rishikesh, Uttarakhand India

${ }^{2-4}$ Department of ENT, All India Institute of Medical Sciences Rishikesh, Uttarakhand, India
}

Corresponding Author: Saurabh Varshney, Professor and Head, Department of ENT, All India Institute of Medical Sciences, Rishikesh-249201, Uttarakhand, India, e-mail: drsaurabh68@gmail.com several centimeters. ${ }^{2}$ Nine other cases of labular capillary hemangioma nasal cavity (LCHNC) have been reported to date in the pediatric population. ${ }^{8}$

In nose most of them are found to be originating from the septum (Kiesselbach area) or vestibule and exceptionally from the inferior and middle turbinates. ${ }^{3}$ Clinical findings usually correspond with a nasal mass that causes a progressive nasal obstruction and intermittent epistaxis. The exact physiopathology of this uncommon entity is unknown, but it is found to be associated with microtrauma (nose picking or nasal packing) and hormonal factors, such as pregnancy and oral contraceptive use. ${ }^{4}$ We present two cases of patients diagnosed and treated for LCH. The clinical features, radiological findings, treatment, and outcomes are discussed.

\section{CASE REPORTS}

\section{Case 1}

A 9-year-old female patient visited our ENT department with a history of 4 months intermittent epistaxis left nose, associated with mild rhinorrhea and left nasal obstruction which was progressive. She had no other clinical symptoms associated and no relevant pathologic history. There was no known history of trauma or recent infection. In the physical examination an irregular, hypervascularized, red-to-purple mass with small ulcerative areas occupied the left nasal cavity, which bled readily on touch. The inferior and middle turbinates were not identifiable in left nose (Fig. 1). A contrast enhanced computed tomography (CECT) scan-nose and paranasal sinuses (PNS) was performed and reported as an enhancing soft tissue mass occupying the left fossa with suspected inferior turbinate origin and no bone erosion (Fig. 2). A complete endoscopic excision with electrocautery of the tumoral base was performed (Fig. 3). Histopathological results informed a dark yellowish elastic tissue fragment with a shiny smooth surface with a $3.0 \times 2.4 \times 1.7$ $\mathrm{cm}$ complete mass diameter suggesting a LCH (Fig. 4). A 6 months postoperative follow-up showed no signs of recurrence and the patient is symptom free.

\section{Case 2}

A 12-year-old male patient reported with a 2 months history of recurrent epistaxis, rhinorrhea and progressive 


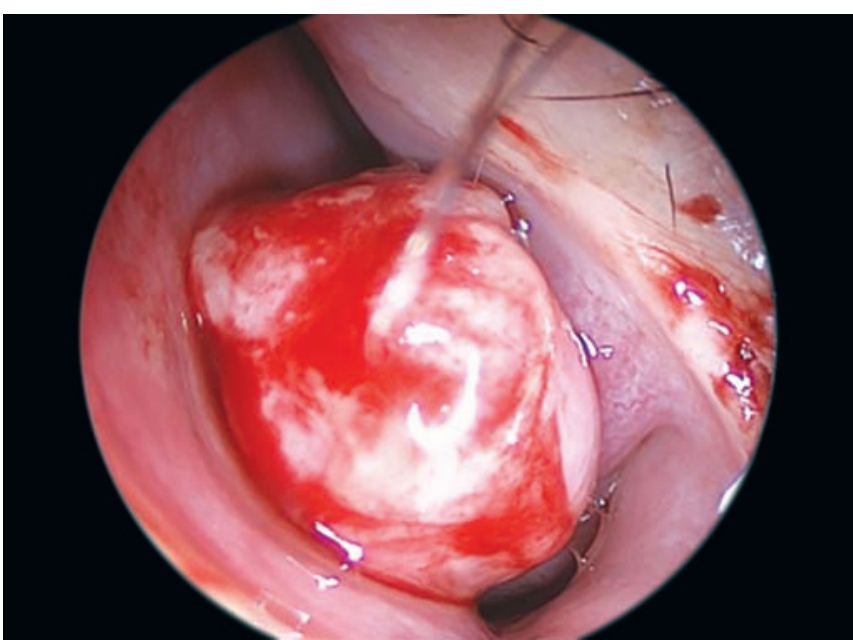

Fig. 1: Nasal endoscopic examination showing an irregular, hypervascularized, red-to-purple mass with small ulcerative areas occupying the left nasal cavity, which bled readily on touch. The inferior and middle turbinates were not identifiable in left nose

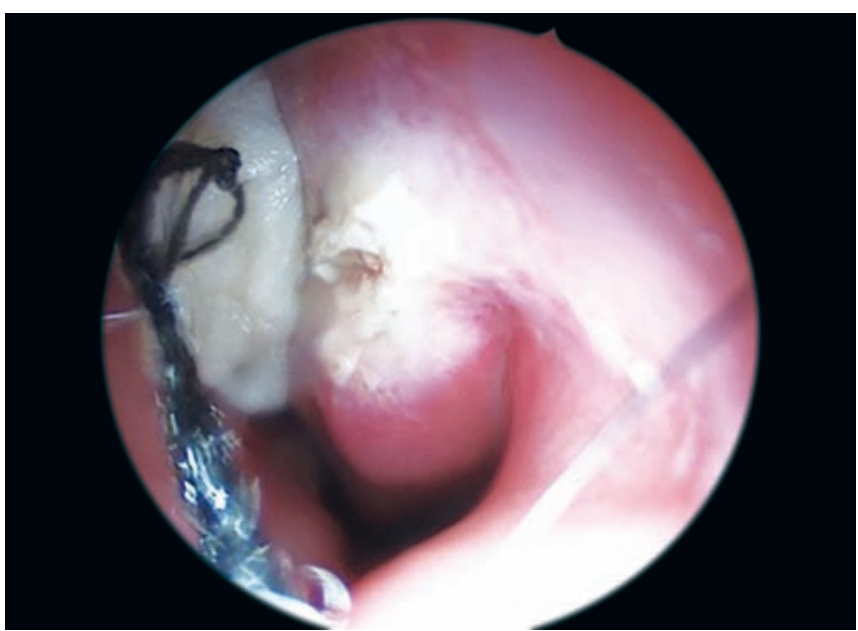

Fig. 3: A complete endoscopic excision with electrocautery of the tumoral base was performed

right nasal obstruction. There was no history of trauma, headache, facial pain, prior surgery or nasal packing for epistaxis. Anterior rhinoscopy and nasal endoscopic examination revealed a red-colored polypoid mass with

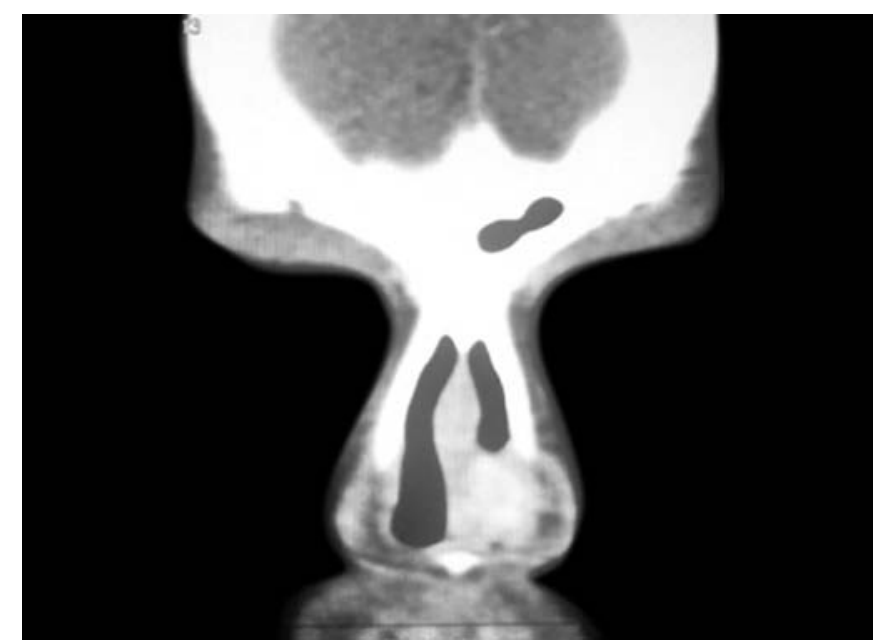

Fig. 2: Coronal section of CT scan, showing enhancing soft tissue mass occupying the left fossa with suspected inferior turbinate origin and no bone erosion (Nose and PNS)

no ulceration occupying the right nasal fossa (Fig. 5). It was seen to be originating from the posterosuperior portion of the septal mucosa and partially obstructing the left nasal passage. No other clinical findings were observed. His blood parameters were within normal limits. A CECT scan-nose and PNS performed revealed a soft tissue mass with strong enhancement with intravenous contrast and no bony erosion with no clear origin established within the right nasal fossa suggesting a hemangioma (Fig. 6). The nasal mass was excised completely under general anesthesia using an electrocautery by endoscopic surgery technique, (which involved partial resection of the mucous septum, the perichondrium and part of mucoperiosteum), with no requirement of preoperative embolization or perioperative blood transfusion. Electrocautery of the base of the tumor was performed observing a septum origin. On histopathology it was a tissue fragment of elastic consistency with a smooth grayish and hemorrhagic surface, of a $3.2 \times 2.2 \times$
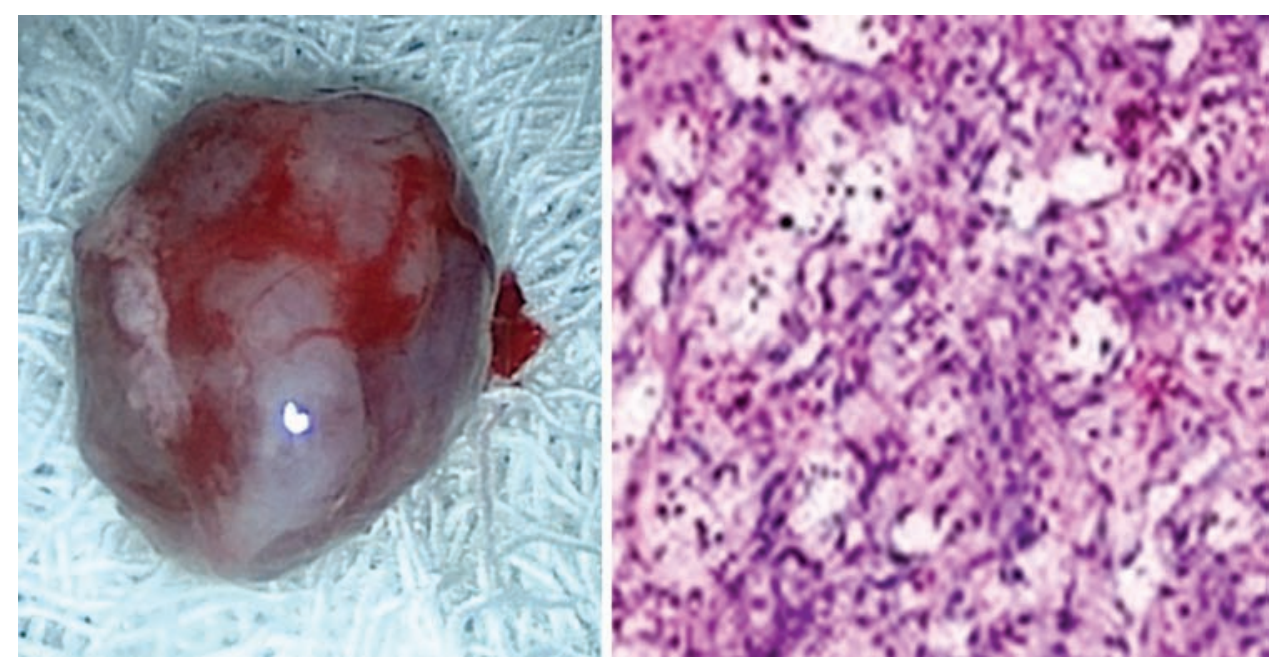

Fig. 4: A $3.0 \times 2.4 \times 1.7 \mathrm{~cm}$ excised mass from left nose, histopathology suggesting a lobular capillary hemangioma 


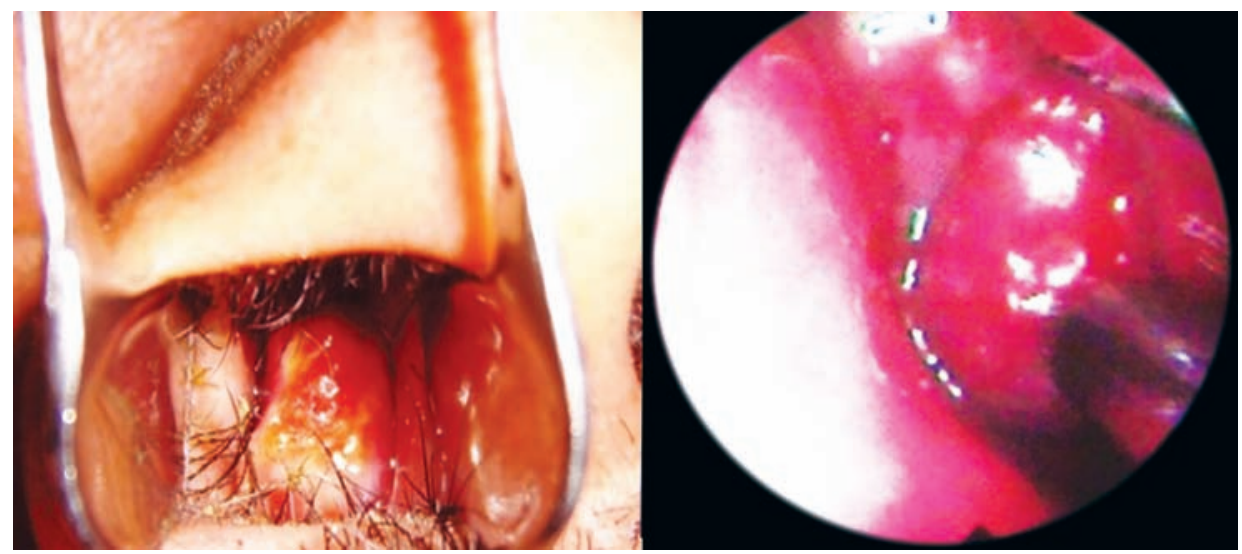

Fig. 5: Anterior rhinoscopy and endoscopic view showing a red-colored polypoid mass with no ulceration occupying the right nasal fossa
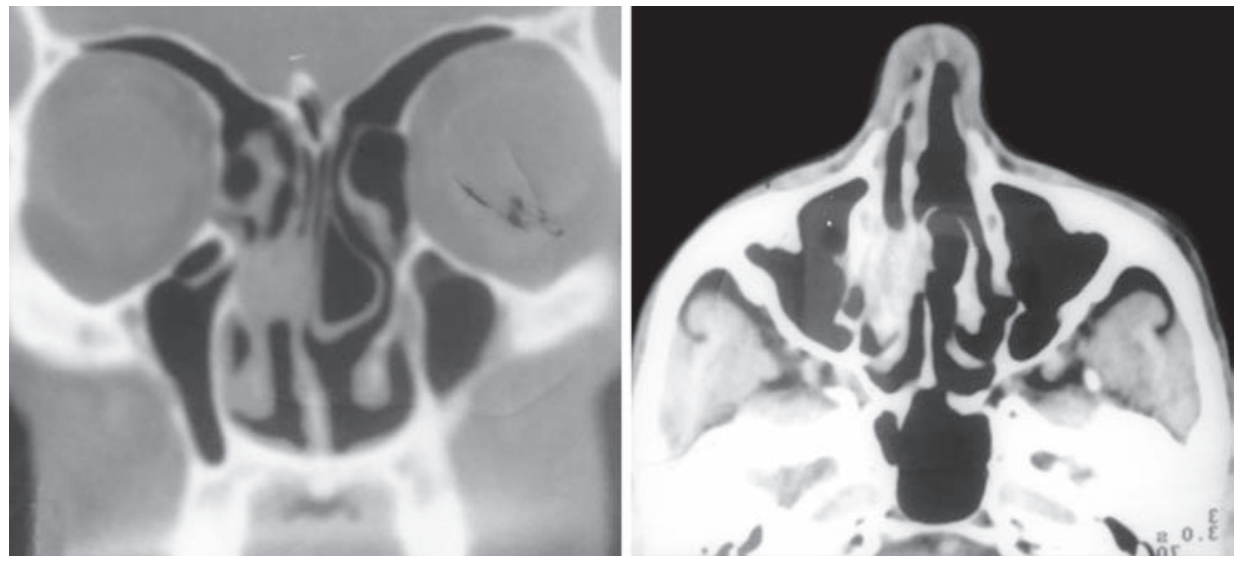

Fig. 6: Contrast-enhanced computed tomography-coronal and axial cuts showing a soft tissue mass with strong enhancement with intravenous contrast and no bony erosion with no clear origin established within the right nasal fossa suggesting a hemangioma

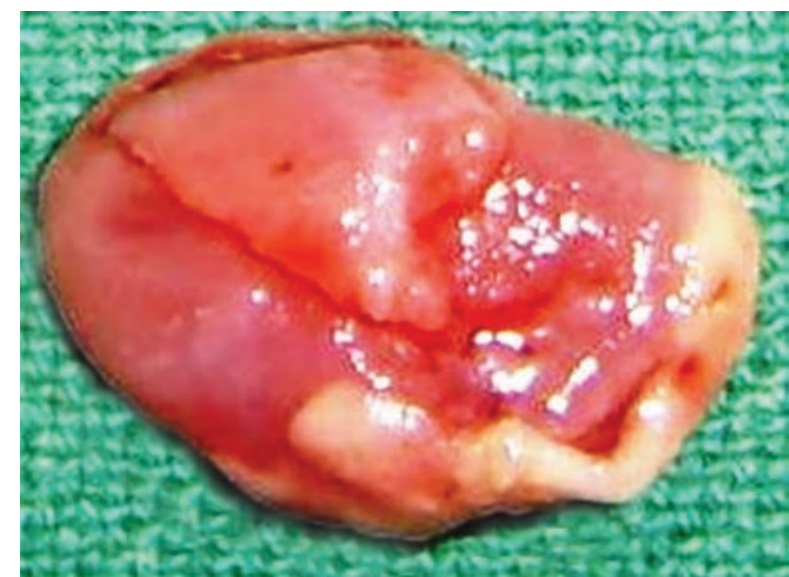

Fig. 7: A $3.2 \times 2.2 \times 1.2 \mathrm{~cm}$ of diameter excised mass suggesting a lobular capillary hemangioma

$1.2 \mathrm{~cm}$ of diameter mass suggesting a LCH (Fig. 7). There were no postoperative complications and a 12 months follow-up showed no signs of recurrence.

\section{DISCUSSION}

Lobular capillary hemangioma is a benign lesion occurring in the skin and mucous membranes. It is considered to be an acquired vascular tumor. ${ }^{6}$

Hemangiomas are classified in various types, such as capillary, cavernous and mixed types that include arterio- venous and epitheliod. The capillary type is mostly found in the skin and oral cavity and in very few occasions in the nasal mucosa. In children they are found to predominate in males but in the adult age they are mostly found in women in the 3 rd to 4 th decade. ${ }^{7}$

In 1897, Poncet and Dor published the first report of 'pea to nut-sized' vascular tumors on the fingers and arms of four patients. The authors referred to this condition as 'human botryomycosis,' speculating that the lesions were secondary to a fungal infection thought to cause morphologically similar lesions in horses. In 1904, Hartzell coined the term 'pyogenic granuloma' to describe these lesions which he presumed to be granulation tissue arising in response to a bacterial infection. ${ }^{8}$ Here, the term is a misnomer as it is neither infectious nor granulomatous. First case of pyogenic granuloma of the nasal cavity was described in 1940 by Frank I, and Blahd M. ${ }^{7}$ In 1980, Mills et al noting the paucity of evidence to support an infectious origin, proposed the term ' $\mathrm{LCH}$ ' derived from the characteristic microscopic features of this tumor. The shifting nomenclature reflects an evolving understanding of the underlying pathogenesis. ${ }^{8}$

Lobular capillary hemangioma is more common in the third decade and in females. ${ }^{6}$ Nasal LCH occurs in children 10 months of age to adults in their 7 th decade. ${ }^{7}$ 
In adults, LCH occurs in as many as 5\% of all pregnant women. ${ }^{8}$ Pagliai and Cohen found that, among 115 children presenting with $\mathrm{LCH}$, more than $60 \%$ were male. The same study identified the most common location of these lesions in children to be the head and neck (76.9\%). Among those patients with head and neck $\mathrm{LCH}$, the majority had lesions on the skin $(36.1 \%$ on the cheek, $12 \%$ on the forehead, $9.6 \%$ on the scalp). Other studies report the frequent appearance of these lesions on the gingiva, lips, tongue, buccal mucosa, and rarely in the nasal cavity. ${ }^{9}$ The most common sites of mucosal $\mathrm{LCH}$ are the gingiva, lips, tongue, and buccal mucosa. Nasal cavity involvement is unusual, with the anterior portion of the septal mucosa and the tip of the turbinate being the most frequently involved areas in the nasal cavity. ${ }^{6}$

The etiology of LCH remains unknown, though there is some evidence to support both trauma and hormonal influences. The relative frequency of $\mathrm{LCH}$ developing at the anterior nasal septum and the anterior aspect of the inferior turbinate, as well as the increased incidence of $\mathrm{LCH}$ in recurrent nose pickers or those with a history of nasal packing, give to the belief that local trauma plays a role in the genesis of $\mathrm{LCH}^{8}$ However, a retrospective study of 112 patients by Pagliai and Cohen found a history of trauma in only five patients (4.5\%) with clinically diagnosed LCH. ${ }^{9}$ Causes have been attributed to hormonal changes, such as pregnancy and contraceptive. ${ }^{5}$ The term pregnancy tumor has been used to reflect the association with pregnancy. ${ }^{10}$ There is a well-established relationship between LCH and pregnancy. Lobular capillary hemangioma commonly occurs in women who are pregnant and those who use oral contraceptives. These lesions regress after delivery, implicating a role of hormones in the growth of $\mathrm{LCH}^{8}$ Viral oncogenes, arteriovenous malformations and angiogenic growth factors are also possible promoting agents. Even rheumatoid arthritis has been described as a possible triggering factor. Increased blood levels of angiogenic growth factors, such as vascular endothelial growth factor (VEGF), and basic fibroblast growth factor (BFGF) may be critical triggering agents. ${ }^{5}$

Clinical findings may include a red-to-purple, hypervascularized mass with possible ulcerated areas. In most reports, they are predominantly found in the Kiesselbach area in the anterior septum. In the largest study of LCH reported in the literature (40 patients) performed by Puxeddu et al in the nasal cavity the nasal septum was predominant, followed by the nasal vestibule, inferior turbinate, middle turbinate and uncinate process. ${ }^{15}$ Lobular capillary hemangioma of the nasal cavity usually presents with recurrent unilateral epistaxis, nasal obstruction, and nasal discharge and rarely with facial pain, alteration of smell and headache. ${ }^{6}$
Diagnostic imaging is imperative. A CECT scan is the first imaging test performed. Computed tomography features of LCHNC consist of an intensely enhancing mass and an iso- or hypoattenuating cap on CECT. The inferior turbinate seems to be a common site of origin, and bony changes are not uncommon features of LCHNC. Computed tomography is useful not only in identifying the site of origin and assessing the extent but also in suggesting the nature of $\mathrm{LCH}^{11}$ Magnetic resonance imaging appearance has not been fully characterized. Marked enhancement of soft tissue mass with a peripheral isointense ring, and a washout time-intensity curve pattern with hyperintensity on T2-weighted images, are characteristic MRI features found in $\mathrm{LCH} .{ }^{12}$

Microscopically it consists of a mass with a lobular growth pattern of capillary proliferation surrounded by fibrous tissue. Two areas are usually distinguished: a lobular deep area and a superficial ulcerative area. The superficial area may undergo secondary changes, such as granulation tissue reaction, stromal edema and capillary dilation. Stroma may be fibromyxoid or edematous with surrounding acute or chronic inflammation. Capillaries are found to be lined by flattened epithelium and filled with blood with surrounding inflammatory infiltrate. ${ }^{13}$ Recently there has been a report of the presence of CD99/p30/p32mic2 cell adhesion glycoprotein intracytoplasmatic dot-like expression pattern in a nasal LCH previously found in tumors, such as anaplastic $\mathrm{T}$ cell lymphoma, pancreatic tumors, colon adenocarcinomas and malignant soft tissue tumors, such as rhabdomyosarcomas between others. ${ }^{14}$

The differential diagnosis of intranasal LCH includes nasal polyp, antrochoanal polyp, meningocele, meningoencephalocele, sarcoidosis, Wegener's granulomatosis, papilloma, Kaposi's sarcoma, hemangiosarcoma, squamous cell carcinoma, and mucosal malignant melanoma. In children, congenital malformations, such as meningoceles, dermoid cysts, angiomatous polyp, schwannoma, angiofibroma, and gliomas should be considered in the differential diagnosis of an intranasal mass and can be differentiated by CT or MRI. ${ }^{8}$

Biopsy is not recommended due to its vascular origin and risk of severe nosebleed. The recommended treatment of $\mathrm{LCH}$ in the nasal cavity is conservative local excision with cautery at the base of the tumor for hemostasis. This technique is associated with low rates of recurrence. Transnasal endoscopic resection is favored over a rhinotomy due to the relatively minimal morbidity of the procedure, the low rate of recurrence, and the better visualization of the tumor and the surrounding anatomy. ${ }^{8}$ Nasal endoscope has been boon to the rhinologist since it not only helps in proper visualization but 
also offers direct facility of treatment to the area that is not accessible. ${ }^{16}$ Excision may be performed with electrocoagulation, cryotherapy, LASER, excisional surgery and excisional surgery following angiography with embolization. In our cases, we performed an endoscopic approach with complete excision and electrocautery at the base of implantation of the tumor without preoperative angio-graphy. Many reports mention its propensity to recur after excision, but the percentage varies depending on the case series and length of follow-up. Low recurrence is estimated if treated with complete excisional surgery. In our cases, there were no recurrences after complete endoscopic resection for more than 6 months of follow-up.

\section{CONCLUSION}

Labular capillary hemangioma is quite a rare lesion of unknown etiology especially when it occurs in the nasal cavity. It should always be considered in the differential diagnosis of vascular lesions within the nasal cavity. Lobular capillary hemangiomas should always be considered as a possible cause of intermittent, painless, epistaxis when a unilateral nasal mass is clinically noticed in the nasal cavity. The treatment of choice for these vascular lesions is complete excision using an endoscopic approach even for large lesions with an excellent prognosis and low rate of recurrence. Awareness of these lesions may help avoiding misdiagnosing them as more aggressive entities which may require more belligerent treatments.

\section{REFERENCES}

1. Akyol MU, Yalciner EG, Doğan AI. Pyogenic granuloma (lobular capillary hemangioma) of the tongue. Int J Pediatr Otorhinolaryngol 2001;58:239-241.

2. Ozcan C, Apa DD, Görür K. Pediatric lobular capillary hemangioma of the nasal cavity. Eur Arch Otorhinolaryngol 2004;261:449-451.
3. Majidi MR, Jafarian AH, Shahabi A. Lobular capillary hemangioma of middle turbinate literature survey and case report. Iran J Otorhinolaryngol 2012 winter;24(66):41-44.

4. Chi $\mathrm{TH}$, Yuan $\mathrm{CH}$, Chien ST. Lobular capillary hemangioma of the nasal cavity: a retrospective study of 15 cases in Taiwan. Balkan Med J 2014;31:69-71.

5. Villarreal IM, Pinilla Urraca M, Garcia H, Martin F, Lopez Cortijo C. Nasal lobular capillary hemangioma: report of two cases and literature review. J Head and Neck Physicians and Surgeons 2014;2(2):2.

6. Kamath PM, Shenoy SV, Kini J, Mukundan A. Lobular capillary hemangioma of the nasal septum: a case report. Egypt J Ear Nose Throat Allied Sci (2014). Available at: http://dx.doi. org/10.1016/j.ejenta.2014.07.004.

7. Patil P, Singla S, Mane R, et al. Nasal lobular capillary hemangioma. J Clin Imaging Sci 2013 Sep 30;3:40.

8. Virbalas JM, Bent JP, Parikh SR. Pediatric nasal lobular capillary hemangioma. Case Reports in Med 2012. Article ID 769630, 4 pages.

9. Pagliai KA, Cohen BA. Pyogenic granuloma in children. Pediat Dermatol 2004;21(1):10-13.

10. Nair S, Bahal A, Bhadauria RS. Lobular capillary hemangioma of nasal cavity. MJAFI 2008;64(3):270-271.

11. Lee DG, Lee SK, Chang HW, Kim JY, Lee HJ, Lee SM, Kwon JH, Woo S. CT Features of lobular capillary hemangioma of the nasal cavity. Am J Neuroradiol 2010;31:749-754.

12. Yang BT, Li SP, Wang YZ, et al. Routine and dynamic MR imaging study of lobular capillary hemangioma of the nasal cavity with comparison to inverting papilloma. Am J Neuroradiol 2013 Nov-Dec;34(11):2202-2207.

13. Smith SC, Patel RM, Lucas DR, et al. Sinonasal lobular capillary hemangioma: a clinicopathologic study of 34 cases characterizing potential for local recurrence. Head Neck Pathol 2013 Jun;7(2):129-134.

14. Handra-Luca A, Abd Elmageed ZY, Gouhier P. CD99 Expression in nasal lobular capillary hemangioma. Histopathol 2014, Apr 4.

15. Roberto P, Marco B, Peppino LG, Giuseppina P, Davide F, Piero N. Lobular capillary hemangioma of the nasal cavity: A retrospective study on 40 patients. Am J Rhinol 2006;20(4): 480-484.

16. Varshney S, Saxena RK. Epistaxis: a retrospective clinical study. Ind J Otolaryngol Head and Neck Surg 2005;57(2): 125-129. 